\title{
An Event-Based SOS for a Language with Refinement*
}

\author{
Arend Rensink \\ Institut für Informatik, University of Hildesheim \\ Hildesheim, Germany
}

In J. Desel, editor, "Structures in Concurrency Theory", Workshops in Computing, pages 294-309. Springer-Verlag, 1995

\begin{abstract}
The notion of action refinement has been studied intensively in the past few years. It is usually introduced in the form of an operator in a process algebraic language, for which a denotational semantics in a suitable model is then given. In this paper we complement this approach by defining a corresponding operational semantics for refinement, in the form of derivation rules for a transition relation. Because of the (well-known) fact that ordinary transition systems are not expressive enough to capture the effects of refinement, we use an event-based transition system model described elsewhere in the literature. The operational semantics of refinement thus defined is equivalent (in fact event isomorphic) to the usual denotational semantics.
\end{abstract}

\section{Introduction}

Process algebras form a well-known specification paradigm for concurrent systems. Typical operators describe such constructions as parallel composition, and such implementation mechanisms as sequential composition. One operator that has been studied in depth in the past six years is action refinement, which basically has the effect of substituting actions in a given behavioural specification with more complex behaviour that in some sense implements those actions. This operator can be seen on the one hand as allowing a top-down design strategy in which activities can first be specified on a very abstract level as single actions and then turned into more concrete, detailed behaviour; and on the other as corresponding to the implementation mechanism of procedure call in declarative languages.

Action refinement has been studied mostly on the basis of constructions on denotational models. Except for a small subclass of refinements (see Czaja et al. [9]), it turns out that the standard model of labelled transition systems does not allow a satisfactory definition: several intuitive properties of refinement such as distribution over parallel composition (without synchronisation) cannot be satisfied. Successful denotational definitions have however been given on several types of partial order models: cf. Van Glabbeek and Goltz [14], Vogler [26], Jategaonkar and Meyer [17], Best et al. [4], Darondeau and Degano [10].

\footnotetext{
*Based on [22, Chapter 3]. This work was initiated while the author was staying at the University of Twente, and is partially supported by the Esprit BRA Project 3096 SPEC (Formal Methods and Tools for the Development of Distributed and Real Time Systems) and the Esprit Working Group 6067 CALIBAN (Causal Calculi Based on Nets)
} 
It should be remarked at this point that there is no general agreement on the question whether refinement should distribute over synchronisation as well (in addition to independent parallel composition). If such distribution is allowed, refinement is interpreted wholly syntactically; this is arguably more faithful to the notion of top-down design, but we know of no models that are compositional with respect to such an operation. The denotational constructions mentioned above, on the other hand, interpret refinement as semantic substitution (in an appropriate model); it does not in general distribute over synchronisation. Goltz, Gorrieri and Rensink have investigated in [15] when these two approaches coincide. In this paper, we adhere to the latter.

The failure of transition systems to model action refinement immediately implies that the standard use of structural operational semantics, where only actions are used as labels, will not work for action refinement. However, several transition system extensions with corresponding operational semantics are known whose expressivity equals that of partial order models, for instance Degano et al. [11], Degano and Gorrieri [12], Boudol and Castellani [6]. On the basis of such extensions it should be possible to give an operational semantics of refinement. One such definition is indeed given in [12].

In this paper we, too, define an operational semantics for refinement, this time on the basis of an approach developed by Langerak [18]: transition labels are extended with event names, derived from annotations that are added to terms of the language before evaluation. This makes for a very smooth extension of the standard semantics, at the cost of the auxiliary machinery for annotation. Refinement can be captured by three operational rules, respectively for the case that the refined action is not yet terminated after a given transition, that it is terminated, and for termination of the term under refinement as a whole. We use the auxiliary concepts of independent transitions and busy refinements, the latter being exactly those action occurrences whose refinement has started but not yet terminated. We claim that the ensuing definition is intuitive and easy to use; we derive some axioms for refinement. As proof of its correctness we compare the semantics with a construction on an event-based model called families of posets, developed by us in [21].

We proceed as follows: Section 2 presents the language under consideration, its standard semantics and its extended semantics using Langerak's event annotations for the fragment without refinement. Section 3 discusses and defines the semantics of the refinement operator. Section 4 presents the corresponding denotational construction and sketches the correctness proof. In Section 5 we discuss related work and draw some conclusions.

\section{Language and semantics}

We consider a language $\mathbf{L}$ generated by the following grammar:

$$
B::=\delta|\varepsilon| a|B+B| B ; B\left|B \|_{A} B\right| B[r] \mid X .
$$

Here $a \in \mathbf{A}$ is an arbitrary action. We use a special pseudo-action $\checkmark \notin \mathbf{A}$ to denote termination; $\mathbf{A}_{\checkmark}:=\mathbf{A} \cup\{\checkmark\}$ is ranged over by $\mu . B_{1}+B_{2}$ denotes choice between $B_{1}$ and $B_{2}$, with neutral element $\delta$ denoting deadlock. $B_{1} ; B_{2}$ is sequential composition, with neutral element $\varepsilon$ denoting termination; it is assumed to bind stronger than + . We also use $\mathbf{L}^{-\varepsilon}$ to denote the fragment 


\begin{tabular}{|c|c|c|}
\hline & & $\begin{array}{l}\vdash \quad a \stackrel{a}{\longrightarrow} \varepsilon \\
\vdash \quad \varepsilon \stackrel{\checkmark}{\longrightarrow} \delta\end{array}$ \\
\hline & $\begin{array}{l}B_{1} \stackrel{\mu}{\longrightarrow} B^{\prime} \\
B_{2} \stackrel{\mu}{\longrightarrow} B^{\prime}\end{array}$ & $\begin{array}{ll}\vdash & B_{1}+B_{2} \stackrel{\mu}{\longrightarrow} B^{\prime} \\
\vdash & B_{1}+B_{2} \stackrel{\mu}{\longrightarrow} B^{\prime}\end{array}$ \\
\hline & $\begin{array}{ll} & B_{1} \stackrel{a}{\longrightarrow} B_{1}^{\prime} \\
B_{1} \stackrel{\checkmark}{\longrightarrow} B_{1}^{\prime} & B_{2} \stackrel{\mu}{\longrightarrow} B_{2}^{\prime}\end{array}$ & $\begin{array}{ll}\vdash & B_{1} ; B_{2} \stackrel{a}{\longrightarrow} B_{1}^{\prime} ; B_{2} \\
\vdash & B_{1} ; B_{2} \stackrel{\mu}{\longrightarrow} B_{2}^{\prime}\end{array}$ \\
\hline$B_{1} \stackrel{\mu}{\longrightarrow} B_{1}^{\prime}$ & $\begin{array}{r}B_{1} \stackrel{a}{\longrightarrow} B_{1}^{\prime} \quad a \notin A \\
B_{2} \stackrel{a}{\longrightarrow} B_{2}^{\prime} \quad a \notin A \\
B_{2} \stackrel{\mu}{\longrightarrow} B_{2}^{\prime} \quad \mu \in A \cup\{\checkmark\}\end{array}$ & $\begin{array}{ll}\vdash & B_{1}\left\|_{A} B_{2} \stackrel{a}{\longrightarrow} B_{1}^{\prime}\right\|_{A} B_{2} \\
\vdash & B_{1}\left\|_{A} B_{2} \stackrel{a}{\longrightarrow} B_{1}\right\|_{A} B_{2}^{\prime} \\
\vdash & B_{1}\left\|_{A} B_{2} \stackrel{\mu}{\longrightarrow} B_{1}^{\prime}\right\|_{A} B_{2}^{\prime}\end{array}$ \\
\hline & $B_{1} \stackrel{\mu}{\longrightarrow} B_{1}^{\prime} \quad \phi(\mu) \stackrel{\lambda}{\longrightarrow} B_{2}^{\prime}$ & $\vdash \quad B_{1}[\phi] \stackrel{\lambda}{\longrightarrow} B_{1}^{\prime}[\phi]$ \\
\hline & $\theta(X) \stackrel{\mu}{\longrightarrow} B$ & $\vdash \quad X \stackrel{\mu}{\longrightarrow} B$ \\
\hline
\end{tabular}

Table 1: Standard operational semantics for the flat fragment of $\mathbf{L}$

of $\mathbf{L}$ without $\varepsilon . \quad B_{1} \|_{A} B_{2}$ is synchronisation of $B_{1}$ and $B_{2}$ over the actions in $A$, where $B_{1} \| B_{2}$ denotes the special case that $A=\varnothing . B[r]$ is refinement of $B$ according to the refinement function $r: \mathbf{A} \rightarrow \mathbf{L}^{-\varepsilon}$ (hence $\varepsilon$ may not be used in refinement terms; the reason for this restriction will be discussed later). Refinement functions are implicitly extended to $r: \checkmark \mapsto \varepsilon$ (this therefore being the only $r$-image in which $\varepsilon$ occurs). Finally, $X \in \mathbf{X}$ is a process name. The meaning of process names is determined by a process environment $\theta: \mathbf{X} \rightarrow \mathbf{L}$ which generates a recursive system of equations.

Except for refinement, all the operators in $\mathbf{L}$ stem from well-known process algebras. Refinement is also known in the restricted case where all the images of $r$ are simple actions: then it is alternatively called a renaming operator and denoted $\phi$. We call a term flat if all instances of refinements are renamings. For the flat fragment of $\mathbf{L}$, the standard operational semantics is given in Table $1 .{ }^{1}$ (Note that the rule for renaming is formulated in a nonstandard way; this is in order to make the generalisation to refinement more direct.)

To obtain a partial order operational semantics, the transition labels of the standard semantics can be enriched with additional information, essentially to encode the causal dependencies among the transitions. The question is in what form this additional information should be provided. Here we apply an approach developed by Langerak [18]. The remainder of this section basically describes the approach, extended only to model sequential composition. Our own contribution, described in the next sections, is the definition of operational rules for refinement, and the choice of denotational model.

First, we replace the action labels by pairs of events and actions. In other words, a transition in our extended semantics will be of the form $B \stackrel{e, \mu}{\longrightarrow} B^{\prime}$, where $e \in \mathbf{E}$ is some arbitrary event. The universe of events $\mathbf{E}$ is assumed

\footnotetext{
${ }^{1}$ Not included are operators for hiding and restriction of actions. The former has been omitted because we are working in a setting where all actions are visible. The latter is implicitly present in the synchronisation operator.
} 


\begin{tabular}{|c|c|c|c|}
\hline & $\begin{array}{l}\vdash \quad{ }_{e} a \stackrel{e, a}{\longrightarrow}(*, e)^{\mathcal{E}} \\
\vdash \quad{ }_{e} \mathcal{E} \stackrel{e, \checkmark}{\longrightarrow} \delta\end{array}$ \\
\hline & & $C_{1} \stackrel{e, \mu}{\longrightarrow} C^{\prime}$ & $\vdash \quad C_{1}+C_{2} \stackrel{e, \mu}{\longrightarrow} C^{\prime}$ \\
\hline & & $C_{2} \stackrel{e, \mu}{\longrightarrow} C^{\prime}$ & $\vdash \quad C_{1}+C_{2} \stackrel{e, \mu}{\longrightarrow} C^{\prime}$ \\
\hline & & $C_{1} \stackrel{e_{1, a}}{\longrightarrow} C_{1}^{\prime}$ & $\vdash \quad C_{1} ; C_{2} \stackrel{e_{1}, a}{\longrightarrow} C_{1}^{\prime} ; C_{2}$ \\
\hline & $C_{1} \stackrel{e_{1}, \checkmark}{\longrightarrow} C_{1}^{\prime}$ & $C_{2} \stackrel{e_{2}, \mu}{\longrightarrow} C_{2}^{\prime}$ & $\vdash \quad C_{1} ; C_{2} \stackrel{e_{2}, \mu}{\longrightarrow} C_{2}^{\prime}$ \\
\hline \multirow{6}{*}{$C_{1} \stackrel{e_{1, \mu}}{\longrightarrow} C_{1}^{\prime}$} & $C_{1} \stackrel{e_{1}}{1}$ & $\stackrel{a}{\rightarrow} C_{1}^{\prime} \quad a \notin A$ & $\vdash \quad C_{1}\left\|_{A} C_{2} \stackrel{\left(e_{1, *}, a\right.}{\longrightarrow} C_{1}^{\prime}\right\|_{A} C_{2}$ \\
\hline & $C_{2} \underline{e_{2}}$ & $\stackrel{a}{\rightarrow} C_{2}^{\prime} \quad a \notin A$ & $\vdash \quad C_{1}\left\|_{A} C_{2} \stackrel{\left(*, e_{2}\right), a}{\longrightarrow} C_{1}\right\|_{A} C_{2}^{\prime}$ \\
\hline & $C_{2} \stackrel{e_{2}, \mu}{\longrightarrow} C_{2}^{\prime}$ & $\mu \in A \cup\{\checkmark\}$ & $\vdash \quad C_{1}\left\|_{A} C_{2} \stackrel{\left(e_{1}, e_{2}\right), \mu}{\longrightarrow} C_{1}^{\prime}\right\|_{A} C_{2}^{\prime}$ \\
\hline & $C_{1} \stackrel{e, \mu}{\longrightarrow} C_{1}^{\prime}$ & $\phi(\mu) \stackrel{d, \lambda}{\longrightarrow} C_{2}^{\prime}$ & $\vdash \quad C[\phi] \stackrel{(e, d), \lambda}{\longrightarrow} C^{\prime}[\phi]$ \\
\hline & & $\theta(X) \stackrel{e, \mu}{\longrightarrow} C$ & $\vdash \quad{ }_{d} X \stackrel{(d, e), \mu}{\longrightarrow} k_{d}(C)$ \\
\hline & & $C \stackrel{e, \mu}{\longrightarrow} C^{\prime}$ & $\vdash \quad k_{d}(C) \stackrel{(d, e), \mu}{\longrightarrow} k_{d}\left(C^{\prime}\right)$ \\
\hline
\end{tabular}

Table 2: Event-based operational semantics for the flat part of $\mathbf{L}$

to be closed under pairing, in order to allow the construction of new events: $(\mathbf{E} \times \mathbf{E}) \cup(\mathbf{E} \times\{*\}) \cup(\{*\} \times \mathbf{E}) \subset \mathbf{E}$, where $* \notin \mathbf{E}$ is a special symbol. These events are generated by annotating the terms of $\mathbf{L}$ according to some scheme, such that all the actions and process names are augmented with a distinguished event. In other words, we do not evaluate $\mathbf{L}$ directly but rather the annotated language $\mathbf{L}(\mathbf{E})$ with the following grammar:

$$
C::=\left.\left.\delta\right|_{e} \varepsilon\right|_{e} a|C+C| C ;\left.C\left|C \|_{A} C\right| C[r]\right|_{e} X \mid k_{e}(C) .
$$

Refinement functions $r$ as well as the process environment $\theta$ now range over $\mathbf{L}(\mathbf{E})$ rather than $\mathbf{L}$. For the operational characterisation of recursive behaviour we need auxiliary operators $k_{e}(C)$ where $k_{e}=\lambda d$. $(e, d)$ is a function $\mathbf{E} \rightarrow \mathbf{E}$ for all $e \in \mathbf{E}$, which glues $e$ to all the event transitions in the execution of $C$, making the events distinct even in infinite behaviour. To ensure that event names do not occur more than once in annotated terms, we restrict ourselves to those terms where the annotation is sound, in the sense that different elements of the term are annotated differently.

It is relatively straightforward to write down an intuitively reasonable operational semantics in the Langerak format for the flat part of $\mathbf{L}(\mathbf{E})$ : see Table 2. Note that in this setup, the images of renaming functions, being a special case of refinement functions, have to be annotated as well.

A sound annotation of a given term $B \in \mathbf{L}$ is easy to construct. For instance, for any "seed" event $e \in \mathbf{E}$, the function $a n n_{e}: \mathbf{L} \rightarrow \mathbf{L}(\mathbf{E})$ defined in Table 3 will correctly annotate $B$. The function strip: $\mathbf{L}(\mathbf{E}) \rightarrow \mathbf{L}$ removes annotations; it should be clear that $\operatorname{strip}\left(\operatorname{ann}_{e}(B)\right)=B$ for all $e \in \mathbf{E}$. Both ann and strip are pointwise extended to refinement functions.

In practice, rather than apply $a n n_{e}$ we will simply enumerate all actions, $\varepsilon s$ and process names in a given term, hence for instance obtaining $\left({ }_{0} a+{ }_{1} \varepsilon\right) ;{ }_{2} b$ from $(a+\varepsilon) ; b$, rather than the much more complex $\left(_{((e, *), *)} a+{ }_{(*,(e, *))} \varepsilon\right) ;_{(*, e)} b$ 


\begin{aligned} & \hline $\operatorname{ann}_{e}(\delta):=\delta \\ & \operatorname{ann}_{e}(B):={ }_{e} B(B \in\{\varepsilon\} \cup \mathbf{A} \cup \mathbf{X}) \\ & \operatorname{ann}_{e}\left(B_{1} \diamond B_{2}\right):=\operatorname{ann}_{(e, *)}\left(B_{1}\right) \diamond \operatorname{ann}_{(*, e)}\left(B_{2}\right)\left(\diamond \in\left\{+, ;, \|_{A}\right\}\right) \\ & \operatorname{ann}_{e}(B[r]):=\operatorname{ann}_{e}(B)\left[\operatorname{ann}_{e}(r)\right] \\ & \operatorname{strip}(\delta):=\delta \\ & \operatorname{strip}\left({ }_{e} B\right):=B(B \in\{\varepsilon\} \cup \mathbf{A} \cup \mathbf{X}) \\ & \operatorname{strip}\left(C_{1} \diamond C_{2}\right):=\operatorname{strip}\left(C_{1}\right) \diamond \operatorname{strip}\left(C_{2}\right)\left(\diamond \in\left\{+, ;, \|_{A}\right\}\right) \\ & \operatorname{strip}(C[r]):=\operatorname{strip}(C)[\operatorname{strip}(r)] \\ & \operatorname{strip}\left(k_{d}(C)\right):=\operatorname{strip}(C) \\ &$\hline \hline\end{aligned}

Table 3: An example annotation function

returned by ann $n^{2}$

The question the becomes how we intend to interpret the operational semantics, and in particular the event labels. It is obvious that different annotations of a given term will result in different transition systems; this difference does not have anything to do with the actual behaviour. To obtain a sensible level of abstraction, we therefore interpret the semantics up to a bijective event renaming.

1 Definition. Two annotated terms $C_{1}, C_{2} \in \mathbf{L}(\mathbf{E})$ are called event isomorphic, denoted $C_{1} \cong_{0} C_{2}$, if there exist a bijection $f: E_{1} \rightarrow E_{2}$ and a relation $\rho \subseteq \mathbf{L}(\mathbf{E}) \times \mathbf{L}(\mathbf{E})$ such that $C_{1} \rho C_{2}$ and for all $C_{1}^{\prime} \rho C_{2}^{\prime}$

- If $C_{1}^{\prime} \stackrel{e, a}{\longrightarrow} C_{1}^{\prime \prime}$ then $e \in E_{1}$ and $\exists C_{2}^{\prime \prime} . C_{2}^{\prime} \stackrel{f(e), a}{\longrightarrow} C_{2}^{\prime \prime} \rho^{-1} C_{1}^{\prime \prime}$;

- If $C_{2}^{\prime} \stackrel{e, a}{\longrightarrow} C_{2}^{\prime \prime}$ then $e \in E_{2}$ and $\exists C_{1}^{\prime \prime} \cdot C_{1}^{\prime} \stackrel{f^{-1}(e), a}{\longrightarrow} C_{1}^{\prime \prime} \rho C_{2}^{\prime \prime}$.

We call the above bisimulation-like relation an isomorphism because for any annotated term $C$, the outgoing event transitions are deterministic:

$$
C \stackrel{e, a}{\longrightarrow} C^{\prime} \wedge C \stackrel{e, a}{\longrightarrow} C^{\prime \prime} \Longrightarrow C^{\prime}=C^{\prime \prime}
$$

This follows from the distinctness of the events in annotated terms. It follows that the event traces of $C$, defined as those strings $\sigma \in(\mathbf{E} \times \mathbf{A})^{*}$ such that $C=C_{0} \stackrel{\sigma_{0}}{\longrightarrow} \stackrel{\sigma_{1}}{\longrightarrow} \cdots$, completely characterise the operational behaviour, and if we denote the set of these traces by $\operatorname{ET}(C)$ then

$$
f: C_{1} \widetilde{\Xi}_{0} C_{2} \Longleftrightarrow E T\left(C_{2}\right)=\left\{f^{*}(\sigma) \mid \sigma \in E T\left(C_{1}\right)\right\}
$$

where $f^{*}$ denotes the pointwise extension of $f$ to event traces. More importantly however, we have

$$
\operatorname{strip}\left(C_{1}\right)=\operatorname{strip}\left(C_{2}\right) \quad \Longrightarrow \quad C_{1} \cong_{0} C_{2}
$$

\footnotetext{
${ }^{2}$ The need to annotate terms before interpreting them makes the semantics noncompositional. A compositional variant is obtained if annotations are generated dynamically by the operational rules, like in the proved transitions of Boudol and Castellani [5]. This does not affect the tenets of this paper; we prefer Langerak's presentation for simplicity.
} 
This implies that we have indeed abstracted from the particular annotation mechanism. Since also for all $B \in \mathbf{L}$ there is a $C \in \mathbf{L}(\mathbf{E})$ such that $\operatorname{strip}(C)=B$ (for instance, $C=\operatorname{ann}_{e}(B)$ ), it follows that the following extension of $\cong_{0}$ to $\mathbf{L}$ is well-defined:

$$
\operatorname{strip}\left(C_{1}\right) \cong_{0} \operatorname{strip}\left(C_{2}\right): \Leftrightarrow C_{1} \cong_{0} C_{2} \text {. }
$$

Event isomorphism is a congruence over $\mathbf{L}(\mathbf{E})$ and through this definition also over $\mathbf{L}$; moreover it subsumes commutativity and associativity of choice, associativity of sequential composition and commutativity of synchronisation; also $\varepsilon$ is a neutral element with respect to sequential composition and $\delta$ with respect to choice. On the other hand, for instance $a ; c+b ; c \not \neq_{0}(a+b) ; c$ and $a ; b+b ; a \neq_{0} a \mid \| b$. Especially the former shows that $\cong_{0}$ is still a very strong notion: it negates the right-distributivity of sequential composition over choice common to almost all equivalences known from the literature. For our purpose this is in fact beneficial, since we will be using $\simeq_{0}$ to show correspondence of the above operational semantics to an event-based denotational semantics; the correspondence will remain valid under any more abstract interpretation than $\widetilde{\Xi}_{0}$, hence the stronger this relation is the better.

A very important question is whether the semantics is in some sense "correct". One immediate observation is that by stripping the terms in Table 2 and removing the event labels from the transitions, we get back Table 1 exactly. In other words, for all annotated terms $C_{1}, C_{2}$ we have

$$
\operatorname{strip}\left(C_{1}\right) \stackrel{a}{\longrightarrow} B_{2} \Leftrightarrow\left(\exists e \in \mathbf{E}, C_{2} \in \mathbf{L}(\mathbf{E}) . C_{1} \stackrel{e, a}{\longrightarrow} C_{2} \wedge \operatorname{strip}\left(C_{2}\right)=B_{2}\right.
$$

This shows that we have directly extended the standard semantics. In fact it is not difficult to prove that $\cong_{0}$ implies isomorphism of the standard semantics derived according to Table 1. A second, more important criterion is the existence of a denotational semantics to go with Table 2 . We return to this issue in Section 4.

\section{Refinement}

Let us discuss the problems involved in extending our operational semantics to refinement. As mentioned in the introduction, we take the traditional view, put forward initially by Aceto and Hennessy [2] and Van Glabbeek and Goltz [13], in which refinement equates to substitution of abstract actions by the concrete behaviour to which they are mapped. For instance, we expect to obtain

$$
B_{1}[r]=(a ; b+(a \| \mid c))\left[a \leadsto a_{1} ; a_{2}\right] \cong_{0} a_{1} ; a_{2} ; b+\left(a_{1} ; a_{2} \| \mid c\right)
$$

where $a \leadsto a_{1} ; a_{2}$ denotes the refinement function mapping $a$ to $a_{1} ; a_{2}$ and all other actions to themselves. In the presence of synchronisation however, it turns out that straightforward syntactic substitution sometimes gives unexpected results; here we follow Van Glabbeek and Goltz in moving to semantic substitution in some sufficiently expressive denotational model (see Section 4). It is this kind of substitution, then, that we wish to capture in operational rules. We aim at rules of the following approximate form:

$$
B_{1} \stackrel{a}{\longrightarrow} B_{1}^{\prime} \quad r(a) \stackrel{b}{\longrightarrow} B_{2}^{\prime} \quad[\cdots] \quad \vdash \quad B_{1}[r] \stackrel{b}{\longrightarrow} B_{1}^{\prime \prime}\left[r^{\prime}\right]
$$

(for the moment disregarding annotations). The important part is choosing representations for $B_{1}^{\prime \prime}$ and $r^{\prime}$. 


\subsection{Basic intuitions}

We take $B_{1}[r]$ in $(3)$ as an example. $B_{1}[r]$ may do $a_{1}$, thereby resolving the choice between the two $a$ 's and moving to either $a_{2} ; b$ or $a_{2}\|\| c$. To denote these behaviours as terms of the form $B_{1}^{\prime \prime}\left[r^{\prime}\right]$, we need to encode which occurrence of $a$ is involved, and the intermediate state of its refinement, which is now at $a_{2}$. Fortunately, a pointer to the individual action occurrences is already available in the form of the annotation. We use these to extend the refinement functions with "busy" refinements. Hence $r$ ' in (4) will extend $r$ by mapping the relevant occurrence of $a$ to $a_{2}$ rather than $a_{1} ; a_{2}$.

The need for a compositional semantics forces us to make a further choice: either the action occurrence involved in the refinement should be removed from the term $B_{1}$ under refinement or it should be left standing. (Note that in particular, we cannot (syntactically) replace the actiona occurrence within $B_{1}$ by the remainder $B_{2}^{\prime}$, since this would not be compositional.) More precisely, $B_{1}^{\prime \prime}$ in (4) equals either $B_{1}^{\prime}$ or $B_{1}$. In (3), if we were to set $B_{1}^{\prime \prime}=B_{1}^{\prime}$ this would yield $B_{1}\left[a \leadsto a_{1} ; a_{2}\right] \stackrel{a_{1}}{\longrightarrow} b\left[r^{\prime}\right]$, after which it is no longer visible that action $b$ depends on the action $a_{2}$ still outstanding. Instead we will use $B_{1}^{\prime \prime}=B_{1}$, and hence $B_{1}\left[a \sim a_{1} ; a_{2}\right] \stackrel{a_{1}}{\longrightarrow}(a ; b+(a \| \mid c))\left[r^{\prime}\right]$. But this causes its own problems, since now it is not clear that the choice in the right hand term between $a ; b$ and $a\|\| c$ has been resolved. Fortunately, here we can use the information in $r^{\prime}$ regarding the "busy refinements"; this tells us which occurrence of $a$ is being refined, hence we can impose a side condition on the rule (4) to ensure that transitions may not be in conflict with busy refinements.

This in turn raises the question how to detect such conflicts. The answer once more lies in the annotations: if a given term may do two transitions with different annotations, those transitions are independent if they do not rule out each other, i.e. if each of them may still occur after the other; otherwise they are in conflict. For instance, if we take an annotated version of $B_{1}$ in (3) then

$$
\begin{array}{ll}
{ }_{0} a ;{ }_{1} b+\left({ }_{2} a\|\|_{3} c\right) & \stackrel{(2, *), a}{\longrightarrow} \stackrel{(*, 3), c}{\longrightarrow} \\
{ }_{0} a ;{ }_{1} b+\left({ }_{2} a\|\|_{3} c\right) & \stackrel{(*, 3), c}{\longrightarrow} \stackrel{(2, *), a}{\longrightarrow}
\end{array}
$$

whereas on the other hand ${ }_{0} a ;_{1} b+\left({ }_{2} a\|\|_{3} c\right) \stackrel{0, a}{\longrightarrow} \underset{(*, 3), c}{\longrightarrow}$. In other words, events $(2, *)$ and $(*, 3)$ are independent whereas 0 and $(*, 3)$ are in conflict. This corresponds to the intuition that if the $a_{1}$-transition of $B_{1}[r]$ is due to ${ }_{0} a$ then $c$ may not occur anymore, whereas $c$ is still allowed if $a_{1}$ is due to ${ }_{2} a$.

\subsection{Auxiliary concepts}

It follows that in order to give operational rules for refinement we need two auxiliary concepts: "busy" refinements and independence (or conflict) between annotations. We will now formalise these concepts.

Intuitively, the outgoing transitions of a given term are independent if they arise out of different and non-synchronising parallel components of that term, and conflicting otherwise. One technique to decide this might be to investigate the internal structure of the events, imparted by the rules for synchronisation in Table 2. This however would be against the notion of abstraction we adhere to, according to which events may be renamed in arbitrary fashion, and hence their 
internal structure cannot hold information. Instead we use the local structure of the transition system to define independence: for all $C \in \mathbf{L}(\mathbf{E})$ and $E \subseteq \mathbf{E}$

$$
C \stackrel{E}{\longrightarrow}: \Leftrightarrow\left(\forall d, e \in E . d=e \vee \exists C^{\prime}, C^{\prime \prime} . C \stackrel{d}{\longrightarrow} C^{\prime} \stackrel{e}{\longrightarrow} C^{\prime \prime}\right)
$$

If $C \stackrel{E}{\longrightarrow}$ we say that the events of $E$ are independent in $C$. For instance, in our running example we have ${ }_{0} a{ }_{1} b+\left({ }_{2} a\|\|_{3} c\right) \stackrel{\{(2, *),(*, 3)\}}{\longrightarrow}$ but ${ }_{0} a ;{ }_{1} b+{ }_{2} a\|\|$ $3^{c) ~} \underset{\{0,(*, 3)\}}{\longrightarrow}$. This definition uses the principle that in event-based models, independence corresponds to confluent diamonds in the state space. Note that in (5) we do not quite test for confluency, since we do not require the end states to coincide. Conceivably one could have $C \stackrel{d}{\longrightarrow} \stackrel{e}{\longrightarrow} C^{\prime}$ and $C \stackrel{e}{\longrightarrow} \stackrel{d}{\longrightarrow} C^{\prime \prime}$ where $C^{\prime} \neq C^{\prime \prime}$. In Section 4, however, we show that such a situation cannot occur in models of $\mathbf{L}$, and that $C \stackrel{E}{\longrightarrow}$ indeed signifies independence of the events in $E$.

To account for intermediate, "busy" refinements, for the purpose of the operational semantics we extend the domain of refinement functions to subsets of $\mathbf{E}$. A refinement function $r$ will henceforth be a function from $\mathbf{A} \cup E$ to $\mathbf{L}(\mathbf{E})$, where $E \subseteq_{\text {fin }} \mathbf{E}$ is the finite set of busy events of $r$, which we will usually denote busy $(r)$. (Infinite sets of busy events cannot come into existence.) Furthermore we will use the following constructions on such extended refinement functions:

$$
\begin{aligned}
r \backslash d & :=r\lceil(\mathbf{A} \cup(\text { busy }(r) \backslash\{d\})) \\
r_{d}^{B} & :=(r \backslash d) \cup\{(d, B)\} .
\end{aligned}
$$

In (6) an event is removed from the set of busy events, effectively throwing away the corresponding residual (presumably because it is terminated); in (7) the image of a busy event is changed, or possibly added if the event was not busy before. Note that $r \backslash d=r$ if $d \notin b u s y(r)$. It follows that busy $(r \backslash d)=$ busy $(r) \backslash\{d\}$ and busy $\left(r_{d}^{B}\right)=$ busy $(r) \cup\{d\}$. Now for convenience we also let such extended $r$ range over annotated actions:

$$
r=\lambda(e, a) \text {. if } e \in \operatorname{busy}(r) \text { then } r(e) \text { else } r(a) \text {. }
$$

In terms of this auxiliary notation, the constructions (6) and (7) can be characterised as follows:

$$
\begin{aligned}
r \backslash d & :=\lambda(e, a) . \text { if } d=e \text { then } r(a) \text { else } r(e, a) \\
r_{d}^{B} & :=\lambda(e, a) . \text { if } d=e \text { then } B \text { else } r(e, a) .
\end{aligned}
$$

To complete the running example of $(3)$, we get $r^{\prime}=r_{d}^{B_{2}^{\prime}}$ where $d=0$ or $d=(2, *)$ depending on the occurrence of $a$, and $B_{2}^{\prime}={ }_{(*, 4)} \varepsilon ;{ }_{5} a_{2}$.

\subsection{Operational rules}

With the necessary preparations out of the way, the actual statement of the operational rules for refinement becomes straightforward: see Table 4 . Note that they reduce to the single rule in Table 2 if $r$ is a renaming function.

The expression $C_{1} \stackrel{\{d\} \cup b u s y(r)}{\longrightarrow}$ tests the independence of the event about to be refined with respect to the existing busy events. Note that for all reachable terms, if $d \in$ busy $(r)$ then $C_{1} \stackrel{\{d\} \cup b u s y(r)}{\longrightarrow}$ is fulfilled automatically (proved by 


\begin{tabular}{|ccccc}
\hline$C_{1} \stackrel{d, a}{\longrightarrow} C_{1}^{\prime}$ & $r(d, a) \stackrel{e, b}{\longrightarrow} C_{2}^{\prime} \stackrel{\checkmark}{\longrightarrow}$ & $C_{1} \stackrel{\{d\} \cup b u s y(r)}{\stackrel{\{}{\longrightarrow}} \vdash C_{1}[r] \stackrel{(d, e), b}{\longrightarrow} C_{1}^{\prime}[r \backslash d]$ \\
$C_{1} \stackrel{d, a}{\longrightarrow} C_{1}^{\prime}$ & $r(d, a) \stackrel{e, b}{\longrightarrow} C_{2}^{\prime} \stackrel{\checkmark}{\longrightarrow}$ & $C_{1} \stackrel{\{d\} \cup b u s y(r)}{\longrightarrow} \vdash C_{1}[r] \stackrel{(d, e), b}{\longrightarrow} C_{1}\left[r_{d}^{C_{2}^{\prime}}\right]$ \\
$C_{1} \stackrel{d, \checkmark}{\longrightarrow} C_{1}^{\prime} \quad r(d, \checkmark) \stackrel{e, \checkmark}{\longrightarrow} C_{2}^{\prime}$ & $C_{1} \stackrel{\{d\} \cup b u s y(r)}{\longrightarrow} \vdash C_{1}[r] \stackrel{(d, e), \checkmark}{\longrightarrow} C_{1}^{\prime}[r]$ \\
\hline
\end{tabular}

Table 4: Operational rules for refinement

induction on the length of the derivation); furthermore if busy $(r)=\varnothing$ then the condition is implied by $C_{1} \stackrel{d, a}{\longrightarrow}$.

Note that the second rule of Table 4 contains a negative premise concerning $\checkmark$-transitions. This could potentially lead to problems of well-definedness; cf. Groote [16]. However, in our system $\checkmark$-transitions can be derived independently from non- $\checkmark$-transitions, and so a stratification in the sense of Groote is immediate. Moreover, due to the fact that refinement functions are restricted to range over $\mathbf{L}^{-\varepsilon}$, if $C$ is derived from an $r$-image then it cannot be the case that both $C \stackrel{\checkmark}{\longrightarrow}$ and $C \stackrel{a}{\longrightarrow}$ (where $a \neq \checkmark)$.

We can now derive the behaviour for our example term (3). Let $r: a \mapsto{ }_{4} a_{1} ;_{5} a_{2}$ and $r: \mu \longmapsto 6 \mu$ for all $\mu \neq a$, and $B={ }_{(*, 4)} \varepsilon ;{ }_{5} a_{2}$; then $r(a) \stackrel{4, a_{1}}{\longrightarrow} B \stackrel{\downarrow}{\longrightarrow}$ and $B \stackrel{5, a_{2}}{\longrightarrow} \stackrel{\checkmark}{\longrightarrow}$ and $r(\mu) \stackrel{6, \mu}{\longrightarrow} \underset{\hookrightarrow}{\longrightarrow}$ for all $\mu \neq a$ and hence

$$
\begin{aligned}
& \left({ }_{0} a ;{ }_{1} b+\left({ }_{2} a\|\|_{3} c\right)\right)[r] \quad \stackrel{(0,4), a_{1}}{\longrightarrow} \quad\left(0 a ;{ }_{1} b+\left({ }_{2} a\|\|_{3} c\right)\right)\left[r_{0}^{B}\right] \\
& \stackrel{(0,5), a_{2}}{\longrightarrow} \quad\left((*, 0) \varepsilon ;{ }_{1} b\right)[r] \\
& \stackrel{(1,6), b}{\longrightarrow} \quad(*, 1) \varepsilon[r] \\
& \left({ }_{0} a ;_{1} b+\left({ }_{2} a\|\|_{3} c\right)\right)[r] \stackrel{((2, *), 4), a_{1}}{\longrightarrow}\left({ }_{0} a ;_{1} b+\left({ }_{2} a\|\|_{3} c\right)\right)\left[r_{(2, *)}^{B}\right] \\
& \stackrel{((2, *), 5), a_{2}}{\longrightarrow} \quad\left((*, 2) \varepsilon\|\|_{3} c\right)[r] \\
& \stackrel{((*, 3), 6), c}{\longrightarrow} \quad((*, 2) \varepsilon \mid \|(*, 3) \varepsilon)[r] \\
& \left({ }_{0} a ;_{1} b+\left({ }_{2} a \mid \|_{3} c\right)\right)[r] \quad \stackrel{((2, *), 4), a_{1}}{\longrightarrow} \quad\left(0 a ;_{1} b+\left({ }_{2} a\|\|_{3} c\right)\right)\left[r_{(2, *)}^{B}\right] \\
& \stackrel{((*, 3), 6), c}{\longrightarrow} \quad\left(2 a \mid \|_{(*, 3)} \varepsilon\right)\left[r_{(2, *)}^{B}\right] \\
& \stackrel{((2, *), 5), a_{2}}{\longrightarrow} \quad\left({ }_{(*, 2)} \varepsilon \|_{(*, 3)} \varepsilon\right)[r] \\
& \left({ }_{0} a ;{ }_{1} b+\left({ }_{2} a \mid \|_{3} c\right)\right)[r] \quad \stackrel{((*, 3), 6), c}{\longrightarrow} \quad\left({ }_{2} a \|_{(*, 3)} \varepsilon\right)[r]
\end{aligned}
$$

It follows that the $\widetilde{0}_{0}$-property in $(3)$ indeed holds. In fact it is not hard to prove the axioms in Table 5, where $i d: \mathbf{A} \rightarrow \mathbf{A}$ is the identity function over $\mathbf{A}$ and $r_{2} \circ r_{1}$ denotes concatenation of refinement functions, defined by $\left(r_{2} \circ r_{1}\right): \mu \mapsto r_{1}(\mu)\left[r_{2}\right]$.

\section{Denotational semantics}

The main tool we have to establish the correctness of the above operational rules is to prove consistency with some denotational semantics. ${ }^{3}$ Event-based

\footnotetext{
${ }^{3}$ In some sense this is the wrong order: one would rather expect a denotational semantics to be measured against an existing operational one. For the case of refinement, however,
} 


\begin{aligned} & \hline \hline$[r]=B \quad(B \in\{\delta, \varepsilon\}) \\ & a[r]=r(a) \\ &\left(B_{1} \diamond B_{2}\right)[r]=B_{1}[r] \diamond B_{2}[r] \quad(\diamond \in\{+, ;, \| \mid\}) \\ & X[r]=\theta(X)[r] \\ &$\hline$B[i d]=B \\ & B\left[r_{1}\right]\left[r_{2}\right]=B\left[r_{2} \circ r_{1}\right] \\ &$\hline\end{aligned}

Table 5: Axioms of refinement

models that can deal appropriately with the flat part of $\mathbf{L}$ are event automata by Pinna and Poigné [20], bundle event structures by Langerak [18] and our own families of lposets [21, 22]. We will use the latter.

A labelled poset (lposet, for short) is a triple $p=\left\langle E_{p}, \leq_{p}, \ell_{p}\right\rangle$ where $E_{p} \subseteq \mathbf{E}$ is a finite set of events, $\leq_{p} \subseteq E_{p} \times E_{p}$ is a partial ordering relation over $E_{p}$, and $\ell_{p}: E_{p} \rightarrow \mathbf{A}_{\checkmark}$ is a labelling function such that $\ell_{p}(e)=\checkmark$ iff $e$ is the unique maximal event of $p$. We denote $A_{p}=\ell_{p}^{*}\left(E_{p}\right)$. The class of labelled posets is denoted LPO. If $p, q \in \mathbf{L P O}$ then $p$ and $q$ are called labelling consistent if $\ell_{p}\left\lceil\left(E_{p} \cap E_{q}\right)=\ell_{q}\left\lceil\left(E_{p} \cap E_{q}\right)\right.\right.$, and $p$ is a prefix of $q$ if $E_{p} \subseteq E_{q}$ is leftclosed according to $\leq_{q}$. The union of labelling consistent lposets is defined by $\bigcup P=\left\langle\bigcup E_{p}, \bigcup \leq_{p}, \bigcup \ell_{p}\right\rangle$. This yields an lposet only under certain conditions, which however will always be fulfilled in this paper.

A family of lposets ( $f p o$, for short) is a nonempty, prefix closed set of labelling consistent lposets. The class of flpos is denoted FPO. A flpo $\mathcal{P}$ is called confluent if for all $p, q \in \mathcal{P}, E_{p}=E_{q}$ implies $p=q$, and coherent if for all $P \subseteq \mathcal{P}, \forall p, q \in P . p \cup q \in \mathcal{P}$ implies $\bigcup P \in \mathcal{P}$.

To interpret flpos we again use event isomorphism: $\mathcal{P}, \mathcal{Q} \in \mathbf{F P O}$ are event isomorphic, denoted $\mathcal{P} \cong_{d} \mathcal{Q}$, if there exists a bijection $f: E_{\mathcal{P}} \rightarrow E_{\mathcal{Q}}$ such that $\mathcal{Q}=\left\{f^{*}(p) \mid p \in \mathcal{P}\right\}$ (where $f^{*}$ is the pointwise extension of $f$ to lposets). We then also denote $\mathcal{Q}=f^{*}(\mathcal{P})$. Note that this relation is defined over flpos while Definition 1 concerns event-labelled transition systems. However, a given flpo $\mathcal{P}$ gives rise to an event-labelled transition system $\operatorname{ETS}(\mathcal{P})=\left\langle\mathcal{P}, p_{\varnothing}, \rightarrow \mathcal{P}\right\rangle$, where $\mathcal{P}$ is the set of states, $p_{\varnothing} \in \mathcal{P}$ is the initial state and $\rightarrow \mathcal{P} \subseteq \mathcal{P} \times \mathbf{E} \times(\mathbf{A} \cup\{\checkmark\}) \times \mathcal{P}$ is a transition relation defined by

$$
p \stackrel{e, \mu}{\mathcal{P}} q: \Leftrightarrow(p \preceq q) \wedge\left(E_{q} \backslash E_{p}=\{e\}\right) \wedge\left(\ell_{p}(e)=\mu\right) .
$$

In addition we use $p \sqrt{ } \mathcal{P}$ to denote $\exists e \cdot p \stackrel{e, \checkmark}{\longrightarrow}$; intuitively $p \checkmark \mathfrak{p}$ means that $p$ is a terminated state of $\mathcal{P}$. There is in general a mismatch between event isomorphism of flpos and of transition systems: although $\mathcal{P} \widetilde{\Xi}_{d} \mathcal{Q}$ implies $\operatorname{ETS}(\mathcal{P}) \cong \operatorname{ETS}(\mathcal{Q})$, the inverse does not hold in general. However, we do have the following:

2 Proposition. If $\mathcal{P}, \mathcal{Q} \in \mathbf{F P O}$ are confluent then $\operatorname{ETS}(\mathcal{P}) \cong_{0} \operatorname{ETS}(\mathcal{Q})$ iff $\mathcal{P} \cong_{d} \mathcal{Q}$.

denotational constructions have been in existence for some time, whereas the operational characterisation is the main contribution of this paper. 
In Section 3 we introduced the notation $C \stackrel{E}{\longrightarrow}$ to express that from state $C$, the events in $E$ may be executed concurrently. The following proposition states that a certain subclass of FPO, including, as we will see, the denotational models of $\mathbf{L}(\mathbf{E})$, this notation indeed has the required meaning.

3 Proposition. If $\mathcal{P} \in \mathbf{F P O}$ is confluent and coherent then for all $p \in \mathcal{P}$ and $E \subseteq \mathbf{E}, p \stackrel{E}{\longrightarrow} \mathcal{P}$ iff there exists a $q \in \mathcal{P}$ such that $p \preceq q, E_{q} \backslash E_{p}=E$ and $E \subseteq \max _{\leq_{q}} E_{q}$. In other words, from the state $p$ the events in $E$ can be executed concurrently, resulting in the state $q$.

We define a denotational semantics $\llbracket \cdot \rrbracket: \mathbf{L}(\mathbf{E}) \rightarrow \mathbf{F P O}$ such that $\operatorname{strip}\left(C_{1}\right)=$ $\operatorname{strip}\left(C_{2}\right)$ implies $\llbracket C_{1} \rrbracket \cong_{d} \llbracket C_{2} \rrbracket$; hence $\llbracket \cdot \rrbracket$ and $\widetilde{\Xi}_{d}$ can be extended to $\mathbf{L}$. The operational and denotational semantics are then proved consistent:

4 Theorem. For all $B_{1}, B_{2} \in \mathbf{L}, B_{1} \widetilde{\Xi}_{0} B_{2}$ if and only if $\llbracket B_{1} \rrbracket \widetilde{\Xi}_{d} \llbracket B_{2} \rrbracket$.

We briefly define the denotational semantics and give a sketch of the proof. First we need a number of additional lposet concepts. If $E_{p} \cap E_{q}=\varnothing$ then the sequential composition of $p$ and $q$ is defined by $p ; q$ where

$$
\begin{aligned}
E_{p ; q} & =\left(E_{p} \backslash \ell_{p}^{-1}(\checkmark)\right) \cup\left\{E_{q} \mid \checkmark \in A_{p}\right\} \\
\leq_{p ; q} & =\leq_{p}\left\lceil\left(E_{p} \cap E_{p ; q}\right) \cup\left(E_{p} \cap E_{p ; q}\right) \times\left(E_{q} \cap E_{p ; q}\right) \cup \leq_{q}\left\lceil\left(E_{q} \cap E_{p ; q}\right)\right.\right. \\
\ell_{p ; q} & =\ell_{p}\left\lceil( E _ { p } \cap E _ { p ; q } ) \cup \ell _ { q } \left\lceil\left(E_{q} \cap E_{p ; q}\right) .\right.\right.
\end{aligned}
$$

To capture synchronisation of terms, for lposets $p$ and $q$ and $E \subseteq\left(E_{p} \cup\{*\}\right) \times$ $\left(E_{q} \cup\{*\}\right)$ we define the (partial) product $p \times_{E} q$ by

$$
\begin{aligned}
E_{p \times E q} & =E \\
\leq_{p \times E q} & =\left\{\left((d, e),\left(d^{\prime}, e^{\prime}\right)\right) \mid d \leq_{p} d^{\prime} \vee e \leq_{p} e^{\prime}\right\}^{*} \\
\ell_{p \times E q} & =\left\{((d, e), \mu) \mid \ell_{p}(d)=\mu \vee \ell_{q}(e)=\mu\right\} .
\end{aligned}
$$

This is only partially defined because the transitive closure of the ordering relation may fail to be antisymmetric. Moreover, we will only use this construct if $E$ A-synchronises $p$ and $q$ for some $A \subseteq \mathbf{A}_{\checkmark}$, which is said to be the case when for all $(d, e) \in E$, either $\ell_{p}(d) \notin \bar{A} \cup\{\checkmark\}$ and $e=*$, or $d=*$ and $\ell_{q}(e) \notin A \cup\{\checkmark\}$, or $\ell_{p}(d)=\ell_{q}(e) \in A \cup\{\checkmark\}$. Finally, we define the refinement of $p$ by a function $w: E_{p} \rightarrow \mathbf{L P O}$ by $w(p)$ where

$$
\begin{aligned}
E_{w(p)} & =\left\{\left(e, e^{\prime}\right) \in E_{p} \times \mathbf{E} \mid e^{\prime} \in E_{w(e)}\right\} \\
\leq_{w(p)} & =\left\{\left(\left(d, d^{\prime}\right),\left(e, e^{\prime}\right)\right) \mid d<_{p} e \vee\left(d=e \wedge d^{\prime} \leq_{w(d)} e^{\prime}\right)\right\} \\
\ell_{w(p)} & =\left\{\left(\left(e, e^{\prime}\right), \mu\right) \mid \mu=\ell_{w(e)}\left(e^{\prime}\right)\right\}
\end{aligned}
$$

This yields an lposet, provided that $w$ behaves well. If $\mathcal{R}: \mathbf{A}_{\checkmark} \rightarrow \mathbf{F P O}$ is given then $w: E_{p} \rightarrow \mathbf{L P O}$ is a $p$-witness of $\mathcal{R}$ if for all $e \in E_{p}, w(e) \mathcal{V}_{\mathcal{R}\left(\ell_{p}(e)\right)}$ if $e$ is non-maximal in $p, w(e) \in \mathcal{R}\left(\ell_{p}(e)\right)$ if $e$ is arbitrary, and $\checkmark \in A_{w(e)}$ implies $\ell_{p}(e)=\checkmark$. Refinement is then extended to flpos by

$$
\mathcal{R}(\mathcal{P})=\{w(p) \mid p \in \mathcal{P}, w \text { a } p \text {-witness of } \mathcal{R}\}
$$

For the construction of infinite behaviour we use the fact that FPO is a complete partial order under $\subseteq$ in which least upper bounds correspond to 


\begin{aligned} & \hline \hline$\llbracket \delta \rrbracket:=\{\langle\varnothing, \varnothing, \varnothing\rangle\} \\ & \llbracket e \varepsilon \rrbracket:=\{\langle\{e\},\{(e, e)\},\{(e, \checkmark)\}\rangle\} \\ & \llbracket e a \rrbracket:=\{\langle\{e, d\},\{(e, e),(e, d),(d, d)\},\{(e, a),(d, \checkmark)\}\rangle\} \quad(d=(*, e)) \\ & \llbracket C_{1}+C_{2} \rrbracket:=\llbracket C_{1} \rrbracket \cup \llbracket C_{2} \rrbracket \\ & \llbracket C_{1} ; C_{2} \rrbracket:=\left\{p ; q \mid p \in \llbracket C_{1} \rrbracket, q \in \llbracket C_{2} \rrbracket\right\} \\ & \llbracket C_{1} \|_{A} C_{2} \rrbracket:=\left\{p \times_{E} q \mid p \in \llbracket C_{1} \rrbracket, q \in \llbracket C_{2} \rrbracket, E\right.$ A-synchronises $\left.p, q\right\} \\ & \llbracket C[r] \rrbracket:=(\lambda a \cdot \llbracket r(a) \rrbracket) \llbracket C \rrbracket \\ & \llbracket e X \rrbracket:=k_{e}^{*}\left(\bigcup_{i \in \mathbb{N}} \llbracket X^{i} \rrbracket\right) \\ & \llbracket k_{e}(C) \rrbracket:=k_{e}^{*}(\llbracket C \rrbracket) \\ &$\hline \hline\end{aligned}

Table 6: Denotational semantics of $\mathbf{L}(\mathbf{E})$

unions, and that the operators above are continuous. Hence the usual fixpoint construction, using standard approximants $X^{i}$, is applicable. The denotational semantics is now summed up in Table 6 . (Note that $k_{e}^{*}(\mathcal{P})$ denotes the application of the event isomorphism $k_{e}=\lambda d$. $(d, e)$ to $\mathcal{P}$.) The following proposition states that this semantics yields confluent and coherent flpos, so that Propositions 2 and 3 are applicable.

5 Proposition. For all $C \in \mathbf{L}(\mathbf{E}), \llbracket C \rrbracket$ is a confluent and coherent flpo.

Hence we have that for all $C_{1}, C_{2} \in \mathbf{L}(\mathbf{E}), \llbracket C_{1} \rrbracket \cong_{d} \llbracket C_{2} \rrbracket$ holds if and only if $E T S \llbracket C_{1} \rrbracket \cong E T S \llbracket C_{2} \rrbracket$. To prove Theorem 4 we therefore only have to show ETS $\| C \rrbracket \cong_{0} C$ for all $C \in \mathbf{L}(\mathbf{E})$. The proof is by induction on the term structure. The most interesting is the case of refinement refinement, which is stated below. The complete proof can be found in [22, Chapter 3].

6 Theorem. For all $C \in \mathbf{L}(\mathbf{E})$ and $r: \mathbf{A} \rightarrow \mathbf{L}(\mathbf{E})$, if $E T S \llbracket C \rrbracket \cong \cong_{0} C$ and $E T S \llbracket r(a) \rrbracket \cong_{0} r(a)$ for all $a \in \mathbf{A}$ then $\operatorname{ETS} \llbracket C[r] \rrbracket \cong_{0} C[r]$.

Proof sketch. We have to define a bijection $f: E_{1} \rightarrow E_{2}$ and a relation $\rho \subseteq$ $\llbracket C[r] \rrbracket \times \mathbf{L}(\mathbf{E})$ such that $p_{\varnothing} \rho C[r]$ and the simulation conditions in Definition 1 are fulfilled. The proof uses the $f_{C}$ and $\rho_{C}$ proving $E T S \llbracket C \rrbracket \cong_{0} C$, and for all $a \in \mathbf{A}$ the $f_{a}$ and $\rho_{a}$ proving $\operatorname{ETS} \llbracket r(a) \rrbracket \cong_{0} r(a)$ (see Definition 1). We denote $\mathcal{R}=\lambda a . \llbracket r(a) \rrbracket, \mathcal{P}=\llbracket C \rrbracket$ and $\mathcal{Q}=\mathcal{R}(\mathcal{P})$. It turns out that in general we can assume that $f_{C}$ and the $f_{a}$ equal the identity over $\mathbf{E}$; this allows us to define $f=i d d_{\mathbf{E}}$ as well. Moreover, it turns out that the relations $\rho_{C} \subseteq \llbracket C \rrbracket \times \mathbf{L}(\mathbf{E})$ and $\rho_{a} \subseteq \mathcal{R}(a) \times \mathbf{L}(\mathbf{E})$ for all $a \in \mathbf{A}$ are injective, which means that we can regard them as functions; we will also construct $\rho$ as a function.

For arbitrary $w(p) \in \llbracket C[r] \rrbracket$ we construct the prefix of $p$ where the witness $w$ is already complete, i.e. on which all $w(e)$ are terminated. By construction this includes at least all the non-maximal events of $p$, but possibly some maximal events as well. Intuitively, the events in $p$ that are not complete are still "busy". We define

$$
\begin{aligned}
\operatorname{busy}(p, w) & :=\left\{e \in E_{p} \mid \neg w(e) \mathcal{V}_{\mathcal{R}\left(\ell_{p}(e)\right)}\right\} \\
\operatorname{cmpl}(p, w) & :=p\left\lceil\left(E_{p} \backslash \operatorname{busy}(p, w)\right) .\right.
\end{aligned}
$$


It follows that $\operatorname{cmpl}(p, w) \preceq p$, and, by Proposition $3, p \stackrel{b u s y(p, w)}{\longrightarrow}$ P. We now construct an extended refinement function $r_{p, w}:(\mathbf{A} \cup E) \rightarrow \mathbf{L}(\mathbf{E})$ where $\operatorname{busy}(r)=E=\operatorname{busy}(p, w)$ : let $r_{p, w}\lceil\mathbf{A}=r$ and for all $e \in \operatorname{busy}(p, w)$

$$
r_{p, w}: e \mapsto \rho_{\ell_{p}(e)}(w(e))
$$

We are now ready to define $\rho$ : for all $w(p) \in \mathcal{Q}$,

$$
\rho: w(p) \mapsto \rho_{C}(\operatorname{cmpl}(p, w))\left[r_{p, w}\right]
$$

This is well-defined because, as mentioned above, $w$ and $p$ are uniquely determined for all $w(p) \in \mathcal{Q}$. If $p=p_{\varnothing}$ then $w=\varnothing$ we get $\operatorname{cmpl}(p, w)=p_{\varnothing}=p$ and $r_{p, w}=r$. It follows that $\rho\left(p_{\varnothing}\right)=\rho_{C}\left(p_{\varnothing}\right)[r]=C[r]$ as required. Due to the functional nature of $\rho$, the simulation properties of Definition 1 collapse to

$$
\begin{aligned}
w(p) \stackrel{(d, e), \mu}{\longrightarrow} \mathcal{Q} w^{\prime}\left(p^{\prime}\right) & \Rightarrow \rho(w(p)) \stackrel{(d, e), \mu}{\longrightarrow} \rho\left(w^{\prime}\left(p^{\prime}\right)\right) \\
\rho(w(p)) \stackrel{(d, e), \mu}{\longrightarrow} C^{\prime}\left[r^{\prime}\right] & \Rightarrow \exists w^{\prime}\left(p^{\prime}\right) \in \rho^{-1}\left(C^{\prime}\left[r^{\prime}\right]\right) \cdot w(p) \stackrel{(d, e), \mu}{\longrightarrow} \mathcal{Q} w^{\prime}\left(p^{\prime}\right)(9)
\end{aligned}
$$

The proof of this naturally divides into three cases, one for each of the operational rules for refinement in Table 4: (1) $\mu \in \mathbf{A}$ and $w^{\prime}(e) \checkmark_{\mathcal{R}(\mu)}$ resp. $e \notin b u s y\left(r^{\prime}\right) ;(2) \mu \in \mathbf{A}$ and $\neg w^{\prime}(e) \checkmark_{\mathcal{R}(\mu)}$ resp. $e \in$ busy $\left(r^{\prime}\right)$; or $(3) \mu=\checkmark$. We sketch the proof of (8) for the first case. From the assumptions it follows that $\operatorname{cmpl}(p, w) \stackrel{d, a}{\longrightarrow} \operatorname{cmpl}\left(p^{\prime}, w^{\prime}\right)$ where $a=\ell_{p^{\prime}}(d)$, and hence $\rho_{C}(\operatorname{cmpl}(p, w)) \stackrel{d, a}{\longrightarrow}$ $\rho_{C}\left(\operatorname{cmpl}\left(p^{\prime}, w^{\prime}\right)\right)$; moreover busy $\left(r_{p, w}\right) \cup\{d\}=E_{c m p l\left(p^{\prime}, w^{\prime}\right)} \backslash E_{c m p l(p, w)}$ and hence $\rho_{C}(\operatorname{cmpl}(p, w)) \stackrel{\{d\} \cup b u s y\left(r_{p, w}\right)}{\longrightarrow}$; finally, $q \stackrel{e, \mu}{\longrightarrow} \mathcal{R}(a) w^{\prime}(d) \sqrt{\mathcal{R}}(a)$ where $q=$ $w(d)$ if $d \in \operatorname{busy}(p, w)$ and $q=p_{\varnothing}$ otherwise; hence $r_{p, w}\left({ }_{d} a\right)=\rho_{a}(q) \stackrel{e, \mu}{\longrightarrow} \stackrel{\checkmark}{\longrightarrow}$. It follows that according to Table $4, \rho(w(p))=\rho_{C}(\operatorname{cmpl}(p, w))\left[r_{w, p}\right] \stackrel{(d, e), \mu}{\longrightarrow}$ $\rho_{C}\left(\operatorname{cmpl}\left(p^{\prime}, w^{\prime}\right)\right)\left[r_{p, w} \backslash d\right]=\rho_{C}\left(\operatorname{cmpl}\left(p^{\prime}, w^{\prime}\right)\right)\left[r_{p^{\prime}, w^{\prime}}\right]=\rho\left(w^{\prime}\left(p^{\prime}\right)\right)$. This concludes the proof.

\section{Conclusions}

On the basis of an approach developed by Langerak in [18], we have defined an event-based operational semantics for a rich process algebraic language $\mathbf{L}$, and extended it with an operator for action refinement. The definition has been proved correct modulo event isomorphism by comparing it with a denotational semantics based on families of posets. The rules for refinement are based on two auxiliary concepts: independence of transitions and intermediate or busy refinements.

\subsection{Related work}

Similar studies can be found elsewhere in the literature. Degano et al. [11] and Boudol and Castellani [6] compare event-based operational and denotational semantics for CCS, which differs from $\mathbf{L}$ in that it has a different form of synchronisation, action prefixing rather than sequential composition, and no refinement. Extending these approaches to sequential composition, especially including the neutral element $\varepsilon$, will cause grave difficulties: for instance, it is 
unclear how to model $\varepsilon$ denotationally in the flow event structures underlying [6]. The extension to refinement may however be less problematic. We conjecture that the concepts of event independence and busy refinements can be translated with relative ease to the operational setting in [6], and our refinement rules may remain essentially unchanged.

Degano and Gorrieri [12] also study operational and denotational semantics for a language with refinement, but with action prefixing and without recursion. The denotational model is again flow event structures. An important difference of our paper with [12] is that correctness there is modulo historypreserving bisimulation, which is weaker than event isomorphism and may in some circumstances be more suitable. Busi, Van Glabbeek and Gorrieri [7] follow the same programme with respect to ST-bisimulation, which is weaker yet. Below we discuss the possibility of characterising weaker equivalences on the basis of our operational semantics.

Aceto and Hennessy deal with refinement syntactically rather than semantically (see the introduction, where we have briefly discussed the syntactic approach, or [15] for an exhaustive comparison of semantic and syntactic refinement). In Section 3 we show that for the synchronisation-free fragment of $\mathbf{L}$ we can likewise interpret refinement syntactically, with results comparable to [2]; in the presence of synchronisation [1] the approaches diverge.

In the introduction we have already listed a number of denotational constructions for refinement; apart from the ones mentioned above however, no corresponding operational semantics has been developed.

\subsection{Evaluation and future work}

An advantage of our approach is the relative ease of proving equivalences between terms on the basis of our operational semantics. For instance, the axioms in Table 5 are straightforward to prove. An interesting test case is

$$
\left(B_{1} \|_{A} B_{2}\right)[r] \equiv\left(B_{1}[r]\right) \|_{A}\left(B_{2}[r]\right) \text {. }
$$

In [15], necessary and sufficient conditions are given under which this is sound for event isomorphism, but the proof, based on denotational constructions, is rather involved. An alternative proof on the basis of the operational semantics in this paper should show a decisive improvement.

The equivalence relation in our correctness criterion, event isomorphism, is rather strong. For instance, it does not satisfy the right distributivity of sequential composition over choice: $(x+y) ; z \neq x ; z+y ; z$ for event isomorphism. For our purpose this is not at all a disadvantage since we use the equivalence exclusively to compare operational and denotational semantics: the stronger the equivalence relation, the stronger this correspondence result. In other circumstances however, a weaker equivalence could be preferable. This is a matter of defining such on the basis of the event-labelled transition system we have used here. For instance, (2) shows that by ignoring the event information in the labels we have access to the entire world of interleaving equivalence. On the other hand, we have given an impossibility result in [23] which shows that weaker event-based equivalences do not preserve global properties like event independence. However, it should be possible to construct a transition system with independence in the sense of Nielsen et al. [19] from a given event-labelled 
transition system, on which the characterisation of equivalences looks more promising.

The event-labelled transition systems we have taken from [18] can be found in many variations in the literature, for instance asynchronous automata $[3,24]$ and trace automata [25]. In Section 2 we have already commented on the possibility of employing a more compositional formalism such as the proved transitions of $[5]$.

\section{References}

[1] L. Aceto and M. Hennessy. Adding action refinement to a finite process algebra. In J. Leach Albert, B. Monien, and M. R. Artalejo, editors, Automata, Languages and Programming, volume 510 of Lecture Notes in Computer Science, pages 506-519. Springer-Verlag, 1991. To apear in Information and Computation.

[2] L. Aceto and M. Hennessy. Towards action-refinement in process algebras. Information and Computation, 103:204-269, 1993.

[3] M. A. Bednarczyk. Categories of Asynchronous Systems. PhD thesis, University of Sussex, Oct. 1987.

[4] E. Best, R. Devillers, and J. Esparza. General refinement and recursion operators for the Petri box calculus. In P. Enjalbert, A. Finkel, and K. W. Wagner, editors, STACS 93, volume 665 of Lecture Notes in Computer Science, pages 130-140. Springer-Verlag, 1993.

[5] G. Boudol and I. Castellani. Permutations of transitions: An event structure semantics for CCS and SCCS. In J. W. de Bakker, W.-P. de Roever, and G. Rozenberg, editors, Linear Time, Branching Time and Partial Order in Logics and Models for Concurrency, volume 354 of Lecture Notes in Computer Science, pages 411-427. Springer-Verlag, 1989.

[6] G. Boudol and I. Castellani. Flow models of distributed computations: Three equivalent semantics for CCS. Rapports de Recherche 1484, INRIA, July 1991. To appear in Information and Computation.

[7] N. Busi, R. van Glabbeek, and R. Gorrieri. Axiomatising ST bisimulation equivalence. In E.-R. Olderog, editor, Programming Concepts, Methods and Calculi, volume A-56 of IFIP Transactions, pages 169-188. IFIP, 1994.

[8] W. R. Cleaveland, editor. Concur '92, volume 630 of Lecture Notes in Computer Science. Springer-Verlag, 1992.

[9] I. Czaja, R. J. van Glabbeek, and U. Goltz. Interleaving semantics and action refinement with atomic choice. In G. Rozenberg, editor, Advances in Petri Nets 1992, volume 609 of Lecture Notes in Computer Science, pages 89-109. Springer-Verlag, 1992.

[10] P. Darondeau and P. Degano. Refinement of actions in event structures and causal trees. Theoretical Comput. Sci., 118:21-48, 1993.

[11] P. Degano, R. De Nicola, and U. Montanari. A partial ordering semantics for CCS. Theoretical Comput. Sci., 75:223-262, 1991. 
[12] P. Degano and R. Gorrieri. An operational definition of action refinement. Technical Report TR-28/92, Università di Pisa, 1992. To appear in Information and Computation.

[13] R. van Glabbeek and U. Goltz. Equivalence notions for concurrent systems and refinement of actions. In A. Kreczmar and G. Mirkowska, editors, Mathematical Foundations of Computer Science 1989, volume 379 of Lecture Notes in Computer Science, pages 237-248. Springer-Verlag, 1989.

[14] R. van Glabbeek and U. Goltz. Refinement of actions in causality based models. In J. W. de Bakker, W.-P. de Roever, and G. Rozenberg, editors, Stepwise Refinement of Distributed Systems - Models, Formalisms, Correctness, volume 430 of Lecture Notes in Computer Science, pages 267-300. Springer-Verlag, 1990.

[15] U. Goltz, R. Gorrieri, and A. Rensink. On syntactic and semantic action refinement. In M. Hagiya and J. C. Mitchell, editors, Theoretical Aspects of Computer Software, volume 789 of Lecture Notes in Computer Science, pages 385-404. Springer-Verlag, Apr. 1994.

[16] J. F. Groote. Transition system specifications with negative premises. Theoretical Comput. Sci., 118:263-299, 1993.

[17] L. Jategaonkar and A. Meyer. Testing equivalences for Petri nets with action refinement. In Cleaveland [8], pages 17-31.

[18] R. Langerak. Transformations and Semantics for LOTOS. PhD thesis, University of Twente, Nov. 1992.

[19] M. Nielsen, V. Sassone, and G. Winskel. Relationships between models for concurrency. In J. W. de Bakker, W.-P. de Roever, and G. Rozenberg, editors, A Decade of Concurrency, volume 803 of Lecture Notes in Computer Science, pages 425-476. Springer-Verlag, 1994.

[20] G. M. Pinna and A. Poigné. On the nature of events. In Mathematical Foundations of Computer Science 1992, Lecture Notes in Computer Science. Springer-Verlag, 1992.

[21] A. Rensink. Posets for configurations! In Cleaveland [8], pages 269-285.

[22] A. Rensink. Models and Methods for Action Refinement. PhD thesis, University of Twente, Enschede, Netherlands, Aug. 1993.

[23] A. Rensink. Order isomorphism does not preserve independence. Bull. Eur. Ass. Theoret. Comput. Sci., 51:228-235, Oct. 1993.

[24] M. W. Shields. Concurrent machines. The Computer Journal, 28(5):449465,1985 .

[25] E. W. Stark. Connections between a concrete and an abstract model of concurrent systems. In M. Main, A. Melton, M. Mislove, and D. Schmidt, editors, Mathematical Foundations of Programming Semantics, volume 442 of Lecture Notes in Computer Science, pages 53-79. Springer-Verlag, 1990.

[26] W. Vogler. Failures semantics based on interval semiwords is a congruence for refinement. In C. Choffrut and T. Lengauer, editors, STACS 90, volume 415 of Lecture Notes in Computer Science, pages 285-297. Springer-Verlag, 1990. 